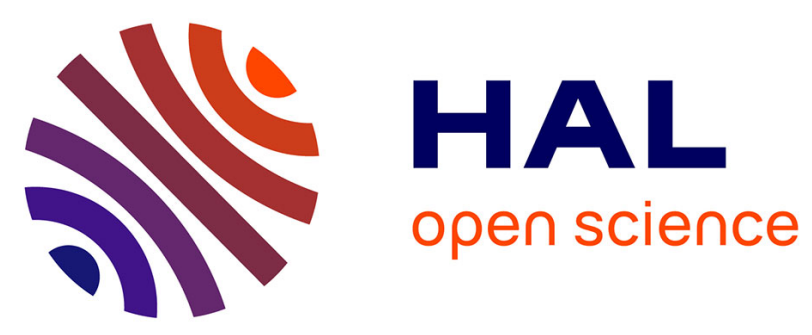

\title{
Amélioration du circuit visuel des contrôleurs aériens pour relier les données entre visualisations en utilisant des transitions animées
}

\author{
Maxime Cordeil, Christophe Hurter, Stéphane Conversy
}

\section{To cite this version:}

Maxime Cordeil, Christophe Hurter, Stéphane Conversy. Amélioration du circuit visuel des contrôleurs aériens pour relier les données entre visualisations en utilisant des transitions animées. IHM 2011, 23ème Conférence Francophone sur l'Interaction Homme-Machine, Oct 2011, Nice, France. pp.Article $\mathrm{N}^{\circ}$ 9, 10.1145/2044354.2044366 . hal-01022274

\section{HAL Id: hal-01022274 \\ https://hal-enac.archives-ouvertes.fr/hal-01022274}

Submitted on 22 Jul 2014

HAL is a multi-disciplinary open access archive for the deposit and dissemination of scientific research documents, whether they are published or not. The documents may come from teaching and research institutions in France or abroad, or from public or private research centers.
L'archive ouverte pluridisciplinaire HAL, est destinée au dépôt et à la diffusion de documents scientifiques de niveau recherche, publiés ou non, émanant des établissements d'enseignement et de recherche français ou étrangers, des laboratoires publics ou privés. 


\section{Amélioration du circuit visuel des Contrôleurs Aériens pour relier les données entre visualisations en utilisant des transitions animées}

\author{
Maxime Cordeil \\ DTI-R\&D/DSNA/ENAC/IRIT \\ 7, Avenue Edouard Belin \\ 31055, Toulouse, France \\ +33 (0) 562259516 \\ cordeil@cena.fr
}

\author{
Christophe Hurter \\ DTI-R\&D/DSNA/ENAC/IRIT \\ 7, Avenue Edouard Belin \\ 31055, Toulouse, France \\ +33(0) 562259576 \\ christophe.hurter@aviation- \\ civile.gouv.fr
}

\author{
Stéphane Conversy \\ Université de Toulouse \\ ENAC \& IRIT \\ Toulouse, France \\ +33 (0) 562174221 \\ stephane.conversy@enac.fr
}

\begin{abstract}
RESUME
Plusieurs affichages sont utilisés dans l'activité du Contrôle du Trafic Aérien, comme la vue radar ODS, la vue des listes de vols et les strips (bandes) papiers. Afin de relier l'information entre ces vues (et ainsi garder le focus sur un sous-ensemble de vols), les contrôleurs effectuent des opérations visuelles (mouvements du regard, analyse des propriétés visuelles etc.). Ce processus peut être coûteux et perturbateur lorsque le trafic augmente et les visualisations affichent un grand nombre d'objets graphiques. Dans cet article nous proposons l'utilisation de transitions animées pour remplacer les circuits visuels empruntés par les contrôleurs. Nous discutons de cette technique et exposons les résultats d'une évaluation prédictive qui suggère une amélioration des performances des utilisateurs.
\end{abstract}

\section{Mots clés}

InfoVis, transitions visuelles, animations, étude de visualisations.

\begin{abstract}
Several separate displays are used by Air Traffic Controllers, such as radar view, flight lists view or paper strips. In order to link the information between these views and keep focus on a subset of flights, controllers are required to do visual operations (eye gaze, analysis of visual properties etc.). This process can be disruptive when traffic increases and when visualizations display large amounts of objects. In this paper we propose the use of animated transitions to replace the visual paths controllers take. We discuss this technique and show results of a predictive evaluation that suggests an improvement in users' performance.
\end{abstract}

\section{Categories and Subject Descriptors}

H5.m. Information interfaces and presentation (e.g., $\mathrm{HCI}$ ): Miscellaneous.

\section{General Terms Design, Human Factors}

Permission to make digital or hard copies of all or part of this work for personal or classroom use is granted without fee provided that copies are not made or distributed for profit or commercial advantage and that copies bear this notice and the full citation on the first page. To copy otherwise, or republish, to post on servers or to redistribute to lists, requires prior specific permission and/or a fee.

IHM'11, October 24-27, 2011, Sophia Antipolis, France

Copyright (C) 2011 ACM 978-1-4503-0822-9/11/10 ...\$10.00.

\section{Keywords}

InfoVis, visual transitions, animations, visualizations study.

\section{INTRODUCTION}

La principale tâche qu'effectuent les Contrôleurs Aériens est le maintien d'une distance minimum de sécurité entre les avions afin de prévenir des conflits. Les données de vol qu'ils manipulent sont multidimensionnelles et peuvent être séparées en deux catégories : les données de l'avion (nom de l'appareil, longitude, latitude, altitude, vitesse etc.) et son plan de vol (les balises qu'il suit, l'heure à laquelle il doit les survoler, le niveau de vol auquel il doit les survoler). Les contrôleurs utilisent différents affichages séparés spatialement pour surveiller ces données et donner des ordres aux pilotes. Pour passer d'un affichage des données de vol à un autre, ils effectuent des transitions visuelles, c'est-à-dire des mouvements du regard pour relier, chercher et retrouver des informations de vol sur lesquelles ils ont le focus. Or, lorsque le trafic augmente, de plus en plus d'objets graphiques sont affichés sur les visualisations rendant ainsi plus complexe la recherche d'ensemble de données. De plus, les données de vols possèdent des représentations différentes selon les affichages; les utilisateurs sont donc amenés à décoder les mêmes informations à travers les différentes visualisations, augmentant ainsi la difficulté pour rechercher des informations dans un contexte de visualisations chargé.

Le travail que nous présentons dans cet article vise à remplacer les transitions visuelles des utilisateurs par des transitions animées gérées par le système. Nous proposons une solution qui consiste à combiner les différentes vues dans un même affichage en n'affichant qu'une seule vue à la fois. Les transitions entre vues sont réalisées par des animations, ce qui permet de minimiser le déplacement du regard et les actions visuelles effectuées par les utilisateurs. Une telle vue, positionnée par exemple sous l'image radar, serait complémentaire à cette dernière. Nous commençons cet article en proposant une taxonomie du support des transitions visuelles. Nous analysons ensuite les trois représentations principales utilisées par les contrôleurs. En nous appuyant sur un scénario d'intégration d'un vol dans un secteur, nous comptons les opérations visuelles élémentaires effectuées par les contrôleurs en utilisant le modèle proposé dans [4], et montrons que la prédiction des performances des utilisateurs est meilleure en utilisant les transitions animées. Nous discutons enfin des intérêts et des limitations de la solution que nous proposons. 


\section{TAXONOMIE DU SUPPORT AUX TRANSITIONS VISUELLES}

Les transitions visuelles permettent aux utilisateurs de relier les données et de garder le focus entre plusieurs représentations [1]. Comme nous l'avons vu, le terme de transition englobe deux concepts liés mais différents ; d'une part il définit les transitions faites par les utilisateurs (c'est l'utilisateur qui « transite » entre les vues), d'autre par il définit les animations pour passer d'une vue ou d'une configuration visuelle à l'autre (c'est la vue ellemême qui effectue les transitions). Nous proposons de classifier les différentes familles de support de transitions visuelles dans deux catégories: les transitions statiques et les transitions dynamiques (ou transitions animées). Dans le cas des transitions statiques, la visualisation ne change pas ; des mécanismes sont mis en œuvre pour permettre à l'utilisateur de garder le focus sur des données et de les relier entre plusieurs vues. Les transitions dynamiques, ou transitions animées, sont une succession des différents états de passage d'une visualisation à une autre dans le temps. Ces transitions permettent de garder le focus sur les données, mais peuvent aussi être un canal supplémentaire pour véhiculer de l'information, pour aider les utilisateurs à comprendre les données. Dans cet article, nous nous intéressons uniquement à l'aspect des transitions qui permet de garder le focus sur les données.

\subsection{Transitions statiques}

Pour effectuer les transitions reliant les informations entre différentes vues, une technique consiste à tracer des traits pour connecter les données correspondant à la même observation. Cette technique est utilisée dans les parallel coordinate plots [1] qui relie des points entre différents scatterplots (nuage de points). Une version étendue est présentée dans FlowViz [9] reliant les points entre scatterplots, les matrices de scatterplots et les plots projetés sur les axes parallèles. Une autre technique permettant le linking entre vues est le painting multiple views [9]. Le principe est d'associer une couleur à un ensemble de données dans un scatterplot, ce qui se répercute dans les autres vues de scatterplots, permettant ainsi à l'utilisateur de suivre les données sur lesquelles il se concentre à travers plusieurs vues. Cette technique est une généralisation du scatterplot brushing [8].

\subsection{Transitions animées}

Heer \& Robertson [5] proposent une taxonomie des changements qui opèrent sur les niveaux syntaxiques et sémantiques des visualisations [6]. En effet, les visualisations peuvent être considérées à un niveau d'analyse syntaxique, qui concerne les variables visuelles [2], et à un niveau sémantique, qui concerne la relation sous-jacente entre les données et leur représentation. Ils proposent de les classer en fonction de la nature des changements (sémantiques ou syntaxiques) dans sept catégories: View Transformation, Substrate Transformation, Filtering, Ordering, Timestep, Visualization Change et Data Schema Change. Ils ont également mené des expérimentations montrant l'efficacité des différentes natures de transitions animées pour le suivi d'objets visuels entre différentes vues.

\subsection{Discussion}

L'utilisation de transitions statiques n'est pas envisageable dans le cas des visualisations utilisées par les contrôleurs car l'ajout de connecteurs (comme des lignes, ou l'utilisation de couleurs pour associer les données) n'est pas compatible avec les visualisations surchargées lorsque le trafic est important. Dans la suite, nous explorons les transitions animées dans le but de relier les données en gardant le focus entre les vues et minimiser les mouvements du regard des utilisateurs.

\section{VISUALISATIONS DU CONTROLE AERIEN}

Les contrôleurs aériens utilisent la vue radar ODS (Ope-rational Display System), un tableau sur lequel sont disposés des strips papiers (contenant la route que suit l'avion dans le secteur) et une vue qui regroupe les avions en fonction de leur point de sortie. Dans cette section nous analysons les informations présentes dans ces visualisations et leur représentation.

\subsection{La vue radar}

La visualisation radar " vue du dessus » des avions évoluant dans un secteur aérien est la principale visualisation utilisée par les contrôleurs. Cette vue contient les routes aériennes, les secteurs et les balises que sont censés survoler les avions (Figure 1, Ecran Radar). Les avions sont représentés par des comètes, qui codent leurs longitudes et leurs latitudes projetées sur les axes $\mathrm{X}$ et $\mathrm{Y}$ de l'écran radar. Ces comètes sont associées à des étiquettes indiquant la vitesse, le nom (ou callsign) et l'altitude de l'appareil. Cette visualisation donne aux contrôleurs une vue d'ensemble du trafic aérien sur un ou plusieurs secteurs.

Tableau 1. Données et représentations de la vue radar

\begin{tabular}{|c|c|}
\hline Données & Représentation \\
\hline Longitude Avion & X Ecran \\
\hline Latitude Avion & Y Ecran \\
\hline
\end{tabular}

\subsection{Les strips papier et le tableau de strips}

Les strips papier constituent une visualisation d'une par-tie du plan de vol de l'avion (Figure 1, Tableau de Strips). Un strip papier est composé d'une partie à gauche qui contient les informations relatives à l'appareil, comme son callsign, son altitude, sa vitesse, le type avion etc... et d'une partie à droite qui représente la route aérienne que suit l'avion. Cette route est une succession de balises. Chaque balise est associée à une heure de passage. Dans certains centres (comme le centre de Bordeaux), les strips papiers sont placés approximativement de façon géographiquement sur le tableau de strip (support rainuré) [7]. Leurs positions sont associées aux positions des avions sur l'écran radar : les contrôleurs font évoluer leur position au grès de l'évolution des comètes sur la vue radar.

Tableau 2. Données et représentations des strips papier

\begin{tabular}{|c|c|}
\hline Données & Représentation \\
\hline$\sim$ Longitude Avion & X Tableau \\
\hline$\sim$ Latitude Avion & Y Tableau \\
\hline
\end{tabular}

\subsection{La vue des flux de sortie}

Les contrôleurs disposent d'une vue dans laquelle sont représentés des mini-strips DYP (Dynamic Presentation) organisés par flux (Figure 1, Fenêtre de Flux) ; les DYPs sont regroupés et ordonnés par niveau de vol et d'heure de sortie dans des fenêtres symbolisant les flux sur les balises de sortie des avions. La position des fenêtres de flux est analogue à la position 
des balises sur l'écran radar. Les strips DYP possèdent des représentations similaires à celles des strips papier mais contiennent le niveau de vol des avions sur les points de sortie.

Tableau 3. Données et représentations des flux de sortie

\begin{tabular}{|c|c|}
\hline Données & Représentation \\
\hline $\begin{array}{c}\text { Latitude Balise } \\
\text { Longitude Balise }\end{array}$ & X Fenêtre \\
Y Fenêtre \\
\hline $\begin{array}{c}\text { Ordre de sortie (niveau } \\
\text { de vol et heure) }\end{array}$ & Y dans la Fenêtre \\
\hline
\end{tabular}

\subsection{Données et représentations partagées}

Ainsi, les visualisations utilisées par les contrôleurs aériens présentent des informations dont certaines sont communes:

- La vue radar affiche la position réelle des avions et les balises. -Les strips dans le tableau de strips sont positionnés de façon analogue au trafic affiché sur l'écran radar.

-Les strips contiennent les informations de l'appareil et sa route. -L'étiquette ODS est une version condensée des strips et la fenêtre des DYP rassemble les strips qui contiennent le niveau de vol des avions sur les balises de sortie.

-Pour chaque visualisation, le niveau de vol, la vitesse au sol et le callsign de l'avion est représenté par du texte.

Les vues sont ainsi connectées par la redondance de l'information; à titre d'exemple, l'utilisateur peut se servir du callsign d'un appareil ou de sa position géographique pour connecter la vue radar et la vue strip.

\section{SCENARIOS ET CIRCUITS VISUELS}

Nous avons mené des observations in situ auprès de contrôleurs aériens (un groupe de contrôleurs en exercice en route, un contrôleur en route, et un contrôleur d'approche) et établi un scénario mettant en évidence un circuit visuel qu'ils effectuent entre les différentes vues. Nous comptons les opérations visuelles élémentaires des utilisateurs en utilisant le modèle proposé dans [4]: seeking/memorizing (recherche et mémorisation d'informations dans une visualisation), entering/exiting (entrer/sortir d'une visualisation), navigating (navigation d'information en information dans une visualisation), predicate \& unpacking (extraction d'information et vérification de prédicat). Dans le scénario suivant, les transitions notées $T i$ font références aux transitions illustrées dans la Figure 1.

\subsection{Circuit visuel du contrôleur organique à l'arrivée d'un avion dans le secteur}

Dix minutes avant l'entrée de l'avion AF3245 dans le secteur de contrôle, un strip est imprimé. Le contrôleur identifie le callsign (T0 entering + seeking), la balise et l'heure de sortie de l'appareil (T1, T2) (seeking + memorizing). Il doit vérifier sur l'écran de flux de sortie si cet avion ne risque pas de provoquer un conflit sur la balise de sortie (LERGA) du secteur. Il repère (T3) la fenêtre de flux de sortie correspondant à la balise $L E R G A$, et compare (T4) les niveaux de vol et les horaires de passage de AF3245 (10:21) et de RAM7154 (10:28) (exiting + navigating + seeking + entering + unpacking + predicate + memorizing $)$. Ces avions passeront au même niveau de vol (350) et à moins de dix minutes sur la même balise $L E R G A$. Il particularise (i.e. associe une couleur) les deux avions. Il regarde (T5, T6) la situation globale du trafic sur son écran radar (exiting + entering + navigating + seeking) et inscrit (T7) l'information de croisement à moins de dix minutes sur la balise $L E R G A$ sur le strip correspondant (exiting + entering + seeking).

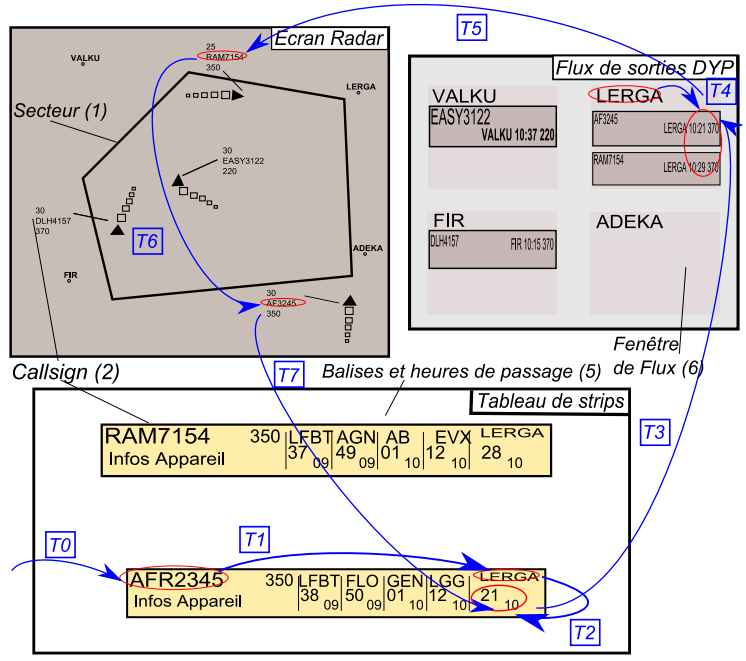

Figure 1. Visualisations utilisées par les contrôleurs et parcours visuel.

Nous comptabilisons donc huit opérations entering/exiting, cinq seeking, deux memorizing, deux navigating et un unpacking \& predicate.

\section{CONCEPTION DES TRANSITIONS}

Le circuit visuel précédent permet d'identifier une transition entre l'écran radar et le tableau de strips et une transition entre le tableau de strips et la fenêtre des DYPs. Nous proposons de combiner les vues dans un seul affichage en utilisant des animations pour les relier. Nous décrivons dans cette section les deux phases de transitions qui interviennent dans le scénario : la transition entre le radar et le tableau de strip et la transition entre le tableau de strip et la fenêtre des DYPs. Pour ce faire, nous considérons dans la suite que le tableau de strips physique est remplacé par un système de stripping électronique, manipulable avec un écran tactile et stylet.

\subsection{Transition Radar ODS $=>$ Tableau de Strip}

Dans cette phase, l'attention du contrôleur est portée sur la comète radar et son strip dans le tableau de strips. Afin de rassembler ces deux objets sous son regard, nous proposons une animation révélant les strips au niveau de la tête de chaîne de la comète (Figure 2, T'1 T'2). La vue finale (Figure 1, Tableau de Strips) de cette transition présente les strips positionnés comme sur le tableau de strips.

\subsection{Transition tableau de strips $=>$ Fenêtre des DYP}

Dans cette phase, le regard du contrôleur est porté sur le strip papier sur lequel il a le focus et sur l'écran des DYPs. L'écran des DYPs organise les strips en fonction de l'heure et du niveau de vol sur leurs balises de sortie. Pour faire la transition entre le tableau de strips et l'écran des DYPs, nous proposons de translater les strips vers leurs points de sortie et d'ajouter le niveau de vol sur les strips dans la case de la dernière balise (Figure 2, T'3 T'4). L'utilisateur ne fait plus que suivre le strip sur lequel il a le focus jusqu'à la balise. A la fin de la transition (Figure 2, Ecran DYP), le regard de l'utilisateur se trouve dans la 
fenêtre des DYP correspondant à la balise de sortie de l'avion, réduisant ainsi la distance entre le tableau de strips et l'écran des DYPs. Il n'a plus qu'à scanner verticalement les strips pour détecter les conflits sur les balises de sorties.

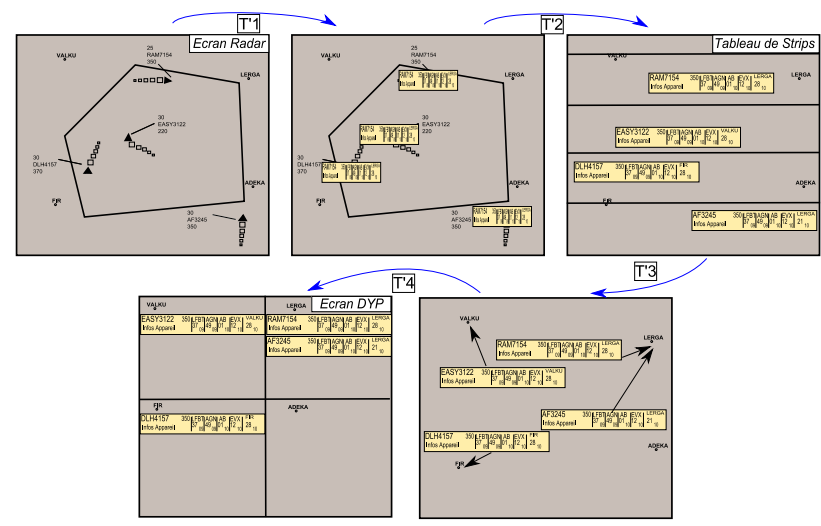

Figure 2. Combinaisons des vues du contrôle aérien. Les flèches illustrent le cheminement des transitions.

Ces transitions permettent d'économiser 7 opérations entering/exiting dans les vues; Les 4 opérations de seeking et les 2 opérations de navigating sont remplacées par des opérations de tracking. En effet, les utilisateurs ne cherchent plus les informations en naviguant dans la vue, mais suivent le déplacement des strips.

\subsection{Intérêts et limitations}

Les transitions conçues montrent un gain de sept actions visuelles de type entering/exiting dans notre scénario. Les actions visuelles de type seeking et navigating sont remplacées par une nouvelle action, le tracking d'objet sur la vue. Cependant, l'utilisation d'animations prend du temps (incompressible et borné), ce qui peut être coûteux pour les utilisateurs connaissant l'emplacement des objets. Dans le cas où les utilisateurs ne connaissent pas l'emplacement des objets, nous formulons l'hypothèse que le tracking d'objets d'intérêt pendant une transition animée sur un même affichage est plus aisé que d'effectuer des transitions visuelles à travers différentes visualisations séparées, lorsque la quantité de données à afficher est élevée.

\section{CONCLUSION ET PERSPECTIVES}

Dans cet article, nous avons présenté la problématique des transitions visuelles entre plusieurs affichages. Nous avons proposé une taxonomie du support des transitions visuelles qui nous permet de comprendre et d'analyser les transitions visuelles dans les visualisations utilisées par les contrôleurs aériens. Nous avons analysé ces visualisations et décrit un circuit visuel en s'appuyant sur un scénario de travail. Nous avons finalement proposé de combiner les différentes vues dans une seule vue en utilisant des transitions animées pour remplacer le circuit visuel des utilisateurs. Nous avons utilisé un modèle théorique permettant d'évaluer de façon prédictive les performances des utilisateurs. Les résultats de cette évaluation suggèrent une amélioration des performances des utilisateurs avec des suppressions d'actions visuelles. Pour étendre et valider ces résultats prédictifs, nous pensons valider l'efficacité de la combinaison de visualisation avec une expérimentation basée sur la recherche d'informations. Nous espérons ainsi montrer expérimentalement que d'une part, la recherche d'information est plus aisée avec des animations, et d'autre part que le temps de recherche est prévisible dans les visualisations combinées.

\section{REMERCIEMENTS}

Nous remercions les membres de l'équipe PII ainsi que les utilisateurs pour leur disponibilité et leur implication dans ces travaux.

\section{REFERENCES}

[1] Andrews, David F. Plots of high-dimensional data. Biometrics, 1972, 28:125-136.

[2] Bertin, J., Graphics and Graphic Information Processing de Gruyter Press, Berlin, (1977).

[3] Buja, A., McDonald, J.A, Michalak, J., and Stuetzle, W. Interactive Data Visualization Using Focusing and Linking. Proc. IEEE Visualization '91, San Di-ego, CA, 1991, pp. 156-163.

[4] Conversy, S., Chatty, S., Hurter, C. Visual Scanning as a Reference Framework for Interactive Representation Design. In Information Visualization, to be published. Sage, 2011.

[5] Heer J. and G. Robertson. Animated Transitions in Statistical Data Graphics. IEEE Trans. on Visualization and Computer Graphics, vol. 13, no. 6, pp. 1240-1247, Nov/Dec, 2007.

[6] Kosslyn S.M. Understanding Charts and Graphs. Applied Cognitive Psychology, 3:185-226. 1989.

[7] Mackay, W. E. (1999). Is paper safer? The Role of paper flight strips in air traffic control. ACM ToCHI. Vol. 6, (4). 311-340.

[8] Newton, C.M. Graphics: from alpha to omega in data analysis. In P.C.C. Wang, editor, Graphical Representation of Multivariate Data. Academic Pres, New York. Proc. of the Symp. On Graphical Representation of Multivariate Data, Naval Postgraduate School, Monterey, Ca., Feb 24, 1978.

[9] Viau, C., McGuffin, M. J., Chiricota, Y., Jurisica, I.. The FlowVizMenu and Parallel Scatterplot Matrix: Hybrid Multidimensional Visualizations for Network Exploration. IEEE Trans. on Visualization and Computer Graphics, Nov./Dec. 2010. 